\title{
Approcci interdisciplinari: letteratura e cartografia. Tra immagini e parole.
}

\author{
Interdisciplinary approaches: literature and cartography. \\ Between images and words.
}

\author{
Fabrizio Di Pasquale \\ Università di Limoges, Francia
}

\begin{abstract}
Riassunto: Nell'ultimo ventennio, un numero importante di lavori sono stati consacrati allo studio della rappresentazione dello spazio nei testi letterari. Tale interesse sembra inscriversi sia nell'evoluzione dei generi, caratterizzati da una spazializzazione crescente delle forme narrative, sia nello sviluppo di pratiche artistiche legate alla creazione di carte letterarie. In seguito all'affermarsi dello spatial turn negli studi letterari e culturali, parte della critica ha focalizzato la sua attenzione sulla relazione che intercorre tra spazio immaginario, spazio referenziale e pratica cartografica. Quest'ultimo aspetto costituisce uno dei temi più interessanti della metodologia geocritica.

II presente articolo mira a studiare questa "convergenza" tra la letteratura e la cartografia, con l'intento di esaminare la testualità delle carte letterarie e, in particolare, la loro dimensione retorica. Le carte letterarie sono in grado di rappresentare i luoghi in cui si svolge l'azione di un romanzo, o di più romanzi, permettendo allo scrittore di costruire un mondo immaginario che i lettori esplorano assieme ai personaggi.
\end{abstract}

Parole chiave: letteratura, cartografia, testo narrativo, geocritica, mapping.

Abstract: For twenty years, a large number of works have been devoted to studying the inclusion of literature in space and the representation of places in literary texts. This interest in space issues seems to fit in both the evolution of literary genres, characterized by an increasing spatialization of narrative forms, and the development of artistic practices related to the creation of literary maps. After what has been termed the spatial turn in literary and cultural studies, critics have focused their attention on the relationships among space, place and mapping in literature. This aspect is one of the most interesting themes in the field of Geocriticism.

This paper aims to study this "convergence" between literature and cartography, examining the textuality of literary maps and, in particular, their rhetorical dimension. Literary maps can represent the story setting, allowing the author to build a fictional world that readers explore with the characters.

Keywords: Literature, Cartography, narrative text, Geocriticism, mapping

\section{Il potere comunicativo delle carte letterarie}

Dal momento in cui la letteratura è stata scritta, la dimensione grafica ha permesso alla temporalità propria al linguaggio di acquisire una certa spazialità'. Parole,

\footnotetext{
1 Bertrand Westphal (2007) mette l'accento sulla natura linguistica e simbolica della carta, nella quale confluiscono diacronia storica e sincronia spaziale.
} 
simboli e colori si sono disposti sulla carta caricandosi di valori estetici. In questo modo, la carta è diventata uno strumento di analisi del testo letterario². Essa ha mostrato, e nel contempo dimostrato, quanto i due elementi che sono il testo e l'immagine siano complementari. Attraverso l'impiego di simboli e di segni specifici, una carta ci consente di costruire un'informazione in un'immagine, di leggerla e semplificarla.

Le regole di questo simbolismo appartengono al campo della semiologia grafica ${ }^{3}$ che stabilisce una sorta di grammatica del linguaggio cartografico. Ciò equivale a riconoscere il messaggio cartografico come un messaggio di interpretazione e di comunicazione scientifica, poiché estende la sua riflessione sulle forme, sui fatti e le relazioni, visibili e invisibili, che sono l'essenza stessa delle realtà geografiche.

La carta è un bel esempio di oggetto ibrido: è la rappresentazione dello spazio fissata nella materia. Questa ibridazione tra la sfera materiale e la sfera ideale è spiegata da Christian Jacob (1992), il quale in L'empire des cartes. Approche théorique de la cartographie à travers l'histoire sembra insistere su due aspetti: la materializzazione e la comunicazione della carta, la materializzazione e la costruzione di un'immagine dello spazio. Materializzazione poiché la carta è un oggetto che può prendere forme differenti, in piano, in rilievo, naturale, artificiale, e che utilizza uno o più materiali; comunicazione poiché c'è sempre un enunciatore e un destinatario che ne interpreta i codici:

[...] reconstruction d'un espace visible ou construction d'un espace invisible, la carte est, dans son processus comme dans son résultat, la projection d'un schéma mental sur un support, la matérialisation d'un ordre intellectuel abstrait de l'univers empirique. (Jacob, $1992: 51$ )

Conformemente alla sua logica analitica, Jacob attribuisce alla carta la funzione di mediazione tra due immagini mentali, quella del suo produttore e quella che conserverà il suo spettatore dopo l'istante della visualizzazione. A tal proposito, nel suo testo Space and place: the perspective of experience (1977), Yi-Fu Tuan ci ricorda che la carta è impiegata per trasmettere in maniera efficace delle conoscenze non soltanto geografiche, per cui sia il creatore che il lettore devono possedere un certo talento per poterne leggere e codificare correttamente valori e significati simbolici:

Perhaps the most common occasion is the need to transmit efficiently geographical knowledge to another person [...]. Cartographic ability presupposes not only a talent for abstraction and symbolization on the part of the primitive cartographer but also a comparable talent in the person who looks on, for he must know how to translate wriggly lines and dots back into real terrain. (Tuan, 1977: 77)

\footnotetext{
2 Le teorie di studiosi come David Harvey (2001), Peter Turchi (2004) e John Pickles (2004) hanno dato alla cartografia un orientamento più umanista.

${ }^{3}$ La semiologia grafica è un campo di interesse della semiotica che si occupa di studiare la trascrizione, il trattamento e la costruzione di immagini grafiche, e più propriamente cartografiche. Secondo Jacques Bertin (1967) la carta è dunque un sistema di segni rigoroso e allo stesso tempo semplice, all'interno del quale possiamo rilevare sei variabili visuali, o anche chiamate grafemi: I'orientazione, la forma, il colore, la superficie, il valore, la taglia. La semiologia grafica è la grammatica della cartografia, essa presuppone diversi livelli di lettura. Oggi la metodologia geocritica prende in esame quello letterario.
} 
Funzione di inventario, funzione di prospettiva, funzione di comunicazione: gli scopi della cartografia sono molteplici. II discorso si fa ancor più complesso man mano che ci addentriamo nell'affascinante mondo della letteratura, laddove la carta può servire allo scrittore a costruire un modello simbolico quantificabile, a disegnare ulteriori percorsi possibili e a suscitare nuove riflessioni.

La domanda di rito resta dunque sempre la stessa: può la cartografia tradurre il significato estetico, geografico e sociologico dei luoghi della rappresentazione letteraria? La risposta è si. Franco Moretti (1997) ha dimostrato d'altronde che le carte letterarie possono leggere lo spazio, con i suoi limiti e i suoi flussi, mettendo in luce la logica interna della narrazione:

\begin{abstract}
Nel frattempo, che cosa ci fanno vedere le carte letterarie? Due cose, direi. Primo, dimostrano la natura ortgebunden, legata al luogo, della letteratura: ogni forma, vedremo, con le sue geometrie, i suoi confini, i suoi tabù spaziali e flussi di movimento. E poi, le carte mettono in luce la logica interna della narrazione: lo spazio semiotico, di intreccio, intorno al quale essa si autoorganizza. La forma letteraria apparirà così come la risultante di due forze contrarie ed egualmente importanti: una esterna e una interna. $\dot{E}$ il problema di sempre, e in fondo il solo vero problema della storia letteraria: la società, la retorica e il loro intrecciarsi. (Moretti, 1997:7)
\end{abstract}

Secondo Franco Moretti, la carta pone sullo stesso piano il pensiero e la vista, consente di operare un transfert, cioè uno spostamento sia fisico che mentale: I am here but I am also elsewhere. A sua volta il romanzo diventa uno spazio privilegiato di proiezione cartografica, quello in cui i luoghi vengono evocati, raccontati, descritti.

Tutti gli elementi del linguaggio cartografico si rivelano particolarmente appropriati per esplorare il potenziale cognitivo della metafora testo-luogo. John Brian Harley (2001) ci ricorda, infatti, che mappe ed atlanti tradiscono la loro immagine di strumenti neutrali per avvicinarsi ad un'idea di prodotto culturale molto simile ad un testo letterario:
Maps cease to be understood primary as inert records of morphological landscapes or passive reflections of the word of objects, but are regarded as refracted images contributing to dialogue in a socially constructed world (...) Maps are never value-free images; except in the narrowest Euclidean sense they are not in themselves either true or false. (Harley, 2001: 53)

A suo modo, John Brian Harley riconosce la possibilità di definire e leggere la carta nei termini di un'epistemologia scientifica: da un lato come un linguaggio iconografico che possiede più livelli di significazione, come aveva già intuito Panofsky 4 (1962), dall'altro come un oggetto della conoscenza, interpretabile attraverso la sociologia del sapere di Foucault.

Egli riconosce che l'immagine cartografica possiede delle leggi proprie di cui l'opera letteraria non si emancipa mai totalmente e, allo stesso tempo, focalizza la sua attenzione sui processi cognitivi e creativi che portano lo scrittore a descrivere,

\footnotetext{
4 Erwin Panofsky individua nelle procedure artistico-geometriche che riguardano lo spazio, anche cartografico, non soltanto l'intenzione della verosimiglianza ma l'espressione effettiva ed effettuante di un contenuto ideologico, filosofico, concettuale, spirituale, storico. Nelle sue teorie la prospettiva diventa forma simbolica.
} 
più o meno simbolicamente, il proprio mondo. Si tratta di un travelling concept che consente di studiare i testi letterari sotto l'angolo della loro referenzialità.

\title{
2. La carta come testo narrativo: il punto di vista geocritico
}

La concezione della carta come testo narrativo, e la conseguente rivalutazione della sua dimensione culturale, costituisce il punto di partenza della metodologia geocritica, la quale considera la cartografia sia come arte e tecnica dell'iscrizione di mappe, sia come poiesis, cioè come consapevolezza critica. Per di più, la cartografia sembra avere una poeticità propria che merita di essere esplorata, così come la poetica degli autori letterari è suscettibile di essere a sua volta cartografata. Non a caso, Bertrand Westphal attualizza il postulato di un'equivalenza semplice ma efficace tra il cartografo e l'autore-artista:

La cartographie transmue en texte et en image une lecture du monde qui jamais n'est univoque. Le cartographe n'est pas une instance anonyme et neutre. C'est un auteur, un artisan, parfois un artiste qui modélise une vision rêvée de l'environnement humain, une manière d'alchimiste qui couvre de dorures des territoires inconnus qui par là même deviennent appétibles. II livre une œuvre, au même titre que l'écrivain. Apparemment, il se fixe l'objectif de couler le monde dans son produit. II vise à le transposer tel quel, selon un référent naturel, alors que l'écrivain, comme tout artiste élaborant une représentation imaginative, le transfigure. Mais, en vérité, le cartographe enclenche une dynamique globale où l'artifice et le naturel s'alimentent I'un l'autre, s'interpénètrent, se confondent quelquefois. [...] C'est que la carte est susceptible d'être lue comme un livre. Et l'atlas est un livre. (Westphal, 2011: 242)

Allo stesso modo Robert Tally Jr. (2013) ci ricorda che la cartografia letteraria ha come compito primario quello di spazializzare la scrittura:

\begin{abstract}
But literary cartography or literary geography, as I understand it, operates precisely by virtue of the specifically literary nature of the project, and writing itself is a form of spatialization that depends upon the reader's acceptance of numerous conventions. Once actual iconographic diagrams or maps are presented, they become supplemental and sometimes competing images to those conjured forth by the narratives themselves. This is also the case with works of criticism or theory. According to Franco Moretti, for example, Mikhail Bakhtin's essay on the chronotope is "the greatest study ever written on space and narrative, and it doesn' $t$ have a single map" (Moretti 2005:35). (Tally, 2013: 5)
\end{abstract}

La cartografia si presenta dunque come dispositivo attraverso cui si esprime una specifica forma di conoscenza-potere, che determina a sua volta una particolare relazione con la realtà, geografica e culturale, che descrive. La narrazione e la carta si configurano come atti linguistici strategici ${ }^{5}$, volti ad assegnare una forma predeterminata allo spazio umano. Ciò equivale a riconoscere sia la validità testuale della mappa, sia il suo potenziale sensoriale nel discorso letterario.

Dalla tradizionale esplorazione dei luoghi della finzione letteraria, la geocritica si è spostata sull'analisi del "senso del luogo", nozione complessa che dipende sia dai

\footnotetext{
${ }^{5}$ Questo aspetto è ben spiegato da Katherine Hayles (2012) per sottolineare il potere del linguaggio tecnologico e informatico. Le moderne carte letterarie utilizzano questo linguaggio facendo appello a sistemi georeferenziati complessi (GIS, Google Maps ecc.).
} 
lineamenti propri di un territorio, che nel loro insieme costituiscono ciò che viene definito "identità del luogo", sia dalla connotazione che tali lineamenti assumono nel vissuto di ciascun individuo. II senso del luogo offre non solo l'opportunità di mettere in primo piano la componente geografica e letteraria, ma anche di riconoscere "l'agire cartografico" nei termini di una pratica culturale attiva, materiale e simbolica, caratterizzata dalla proiezione di specifici marchi antropici e semiotici.

Per Bertrand Westphal, il linguaggio cartografico è in grado di rappresentare la complessità di un romanzo: il grado di importanza dei personaggi, la relazione affettiva e simbolica che il narratore stabilisce con i luoghi descritti, e persino le dinamiche dell'intreccio.

In Atlante del romanzo europeo (1800-1900), ad esempio, Franco Moretti riesce a cartografare i luoghi geografici descritti nei romanzi di Sir Arthur Conan Doyle e Jane Austen, con l'obiettivo di distinguere la funzionalità degli spazi urbani e rurali all'interno della narrazione, oppure a visualizzare la struttura profonda de I promessi sposi di Alessandro Manzoni, nonché la relazione che intercorre tra i personaggi e la società in cui si muovono. Si tratta, in ogni caso, soltanto di alcuni dei numerosi esempi proposti dall'autore nel suo testo, esempi che ci consentono di applicare la cartografia a qualsiasi genere letterario.

\section{Brevi esempi di opere geografiche}

Le carte letterarie sono in grado di descrivere i luoghi in cui si svolge l'azione di un romanzo, o di più romanzi, permettendo allo scrittore di costruire un mondo immaginario che i lettori esplorano assieme ai personaggi. Numerosi sono gli scrittori che, già in passato, hanno sperimentato la fusione tra letteratura e cartografia. Si pensi, ad esempio, alla mappa dell'Isola del tesoro di Robert Louis Stevenson, indispensabile per comprendere la concatenazione degli eventi narrativi, oppure a quella de II signore degli anelli di John Ronald Revel Tolkien, senza la quale è impossibile seguire le avventure della Compagnia dell'Anello nella Terra di mezzo: ad Eriador, nel Bosco Atro e a Mordor.

Lo stesso vale per la geografia letteraria della Contea del Wessex, in cui Thomas Hardy ridisegna la regione del Dorset per ambientarvi racconti e poemi, e quella di Yoknapatawpha dello scrittore americano William Faulkner, lvogo immaginario in cui si fondono mito e realtà, storia e discorso sociale. Agli albori del genere fantastico troviamo anche il mondo delle Cronache di Narnia di Clive Staples Lewis, abitato da misteriose creature, principi e streghe, e quello di Le cronache del ghiaccio e del fuoco di George Raymond Richard Martin, diviso in due regni, Westeros a occidente ed Essos ad oriente.

Si tratta di opere geografiche che si sono costruite in un certo qual modo intorno ad un percorso cartografico. Ce ne sono altre, invece, che dando particolare rilievo alla componente spaziale si prestano naturalmente a questo tipo di pratica. Ne La divina commedia, ad esempio, il percorso morale e spirituale del Dante-narratore passa allegoricamente per l'Inferno, il Purgatorio e il Paradiso, dei quali l'autore offre tre cartografie immaginarie differenti. L'Inferno è la cantica concepita con maggior rigore geometrico, il Purgatorio è formato da luoghi contrastanti e indistinti, mentre il Paradiso manca di uniformità e gradualità tipici dei paesaggi. 
Immaginari o reali, tutti i luoghi della rappresentazione letteraria, indipendentemente dall'autore e dal genere di appartenenza, possono essere cartografati. II romanzo di Jules Verne II giro del mondo in ottanta giorni offre uno spunto interessante, laddove all'elemento del viaggio avventuroso si unisce l'interesse enciclopedico per le scienze applicate e per la geografia, per popoli e paesi Iontani dalla cultura europea. Gli itinerari di Phileas Fogg e Passepartout arricchiscono l'immaginazione del lettore, proponendo una vasta gamma di scenari e luoghi facilmente cartografabili.

Un ulteriore tentativo potrebbe riguardare il romanzo I miserabili di Victor Hugo. Tra fughe e nascondigli, delusioni e amori non corrisposti, sacrifici e redenzione, la storia è ambientata nella Parigi degli anni trenta dell'Ottocento. Una carta letteraria permetterebbe di ripercorrere la ville lumière sulle tracce dei Miserabili, nei luoghi che hanno ispirato Victor Hugo, dove finzione letteraria e realtà si sovrappongono: da Place des Vosges a Place de la Bastille, da Rue Plumet a Rue Saint-Denis, da Rue de Sévigné alla chiesa Saint-Paul Saint-Louis. I luoghi descritti, con la loro dimensione popolare e tragica, fanno da sfondo al più grande affresco sociale delle Francia postnapoleonica, quella tra il 1815 e il 1833. Lo spazio e il tempo troverebbero cosí, nella rappresentazione cartografica, una loro dimensione simbolica, generando infinite possibilità di approfondimento.

Non sono da meno I malavoglia e II Mastro-don Gesualdo di Giovanni Verga, nei quali l'autore "cartografa" la Sicilia del XIX secolo attraverso gli spostamenti e i comportamenti dei suoi personaggi, facendo di quest'ultima un oggetto di indagine storica. I luoghi descritti, Vizzini, Catania, Aci Trezza, assurgono a metafora della società stessa, tra progresso e tradizione, ricchezza e povertà. Essi conferiscono uno spessore caratteriale ed una profondità tale ai personaggi da renderli metaforicamente "animati di vita propria".

Le possibilità di cartografare le opere letterarie sono molte, cosí come gli universi da scoprire. Lo scopo è di riscrivere una storia con gli strumenti del viaggiatore, di ieri e di oggi: la carta geografica, la guida turistica e persino il navigatore satellitare. L'analisi di un romanzo, un poema o un racconto, diventa dunque particolarmente stimolante se effettuata attraverso le coordinate di spazio, tempo e mentalità, nel qui e nell'ora.

Gli esempi in letteratura non mancano, per cui gli autori citati fino ad ora costituiscono solo una minima parte di quelli ai quali si potrebbe applicare uno studio cartografico. Essi ci consentono di mettere in evidenza il ruolo della letteratura come produttrice di nuove cartografie. Non bisogna dimenticare, infatti, che la percezione e la conoscenza dei luoghi non avviene soltanto con il contatto diretto con il mondo reale, ma anche con le rielaborazioni mentali fornite da narratori e poeti.

\section{Conclusioni}

Navigando nel mare della letteratura ci accorgiamo che romanzieri, artisti e poeti non sono esenti dalle pratiche cartografiche. Viaggi e luoghi geografici sono stati da sempre raccontati attraverso le parole, parlate e scritte, di scrittori che hanno cercato di interpretare la realtà suggerendo a loro modo un immaginario cartografico. 
Tentare di cartografare un romanzo significa costruire, ricostruire, immaginare, ricreare, persino distorcere o manipolare, il mondo dell'autore secondo dei codici simbolico-grafici capaci di generare connessioni imprevedibili, percorsi inaspettati, quindi storie e narrazioni.

A metà strada tra codice iconografico e narratività, le carte letterarie sono piani d'azione dall'elevato potenziale immaginativo in grado di suggerire istanze critiche, ideologiche ed esistenziali. D'altra parte, è difficile leggere un'opera letteraria senza immaginare viaggi, attraversamenti e incontri. Si attraversano mondi per coglierne le atmosfere e le intensità emotive, e questo desiderio è da sempre presente nelle pratiche artistiche.

La carta letteraria costituisce, in tal senso, una sorta di guida per il lettore nello studio di questi mondi immaginari, uno strumento di analisi al tempo stesso specifico e mobile, in grado di proporre nuove chiavi di lettura del rapporto che il narratore e i suoi personaggi istaurano con lo spazio.

\section{Bibliografia}

BERTIN, J. (1967): Sémiologie graphique. Les diagrammes, les réseaux, les cartes. Paris: La Haye, Mouton.

BROSSEAU, M. (1996) : Des romans-géographes. Paris: L'Harmattan.

HAYLES, K. N. (2012): How we think. Digital media and contemporary technogenesis. Chicago: The University of Chicago Press.

HARLEY, J. B. (2001): The new nature of maps. Baltimore; London: The Johns Hopkins University Press.

HARVEY, D. (2001): Cartographic identities: geographical knowledges under globalization. In Spaces of capital: towards a critical geography. Edinburgh: Edinburgh University Press. Pp.208-233.

JACOB, C. (1992) : L'empire des cartes. Approche théorique de la cartographie à travers l'histoire. Paris: Albin Michel.

MORETTI, F. (1997): Atlante del romanzo europeo 1800-1900. Torino: Einaudi.

- (2005): Graphs, maps, trees. Abstract models for a literary theory. New York; London: Verso.

PANOFSKY, E. (1962): Il significato nelle arti visive. Torino: Einaudi.

PICKLES, J. (2004): A history of spaces: cartographic reason, mapping and the geocoded world. London: Routledge.

TALLY JR. R. (2013): Spatiality. The new critical idiom. New York: Routledge.

TUAN, Y. F. (1977): Space and place: the perspective of experience. Minneapolis: University of Minnesota Press.

TURCHI, P. (2004): Maps of the imagination. The writer as cartographer. San Antonio; Texas: Trinity University Press. 
WESTPHAL, B. (2007) : La Géocritique, réel, fiction, espace. Paris: Les Éditions de Minuit.

- (2011) : Le monde plausible. Espace, lieu, carte. Paris: Les Éditions de Minuit.

\section{Alcune mappe citate nel testo}

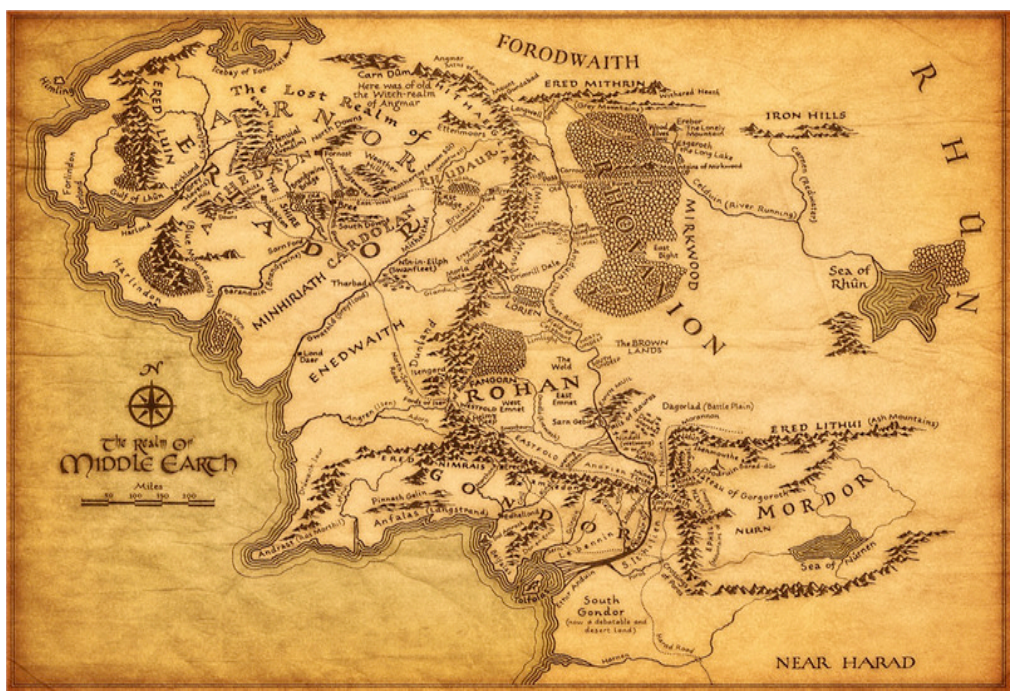

Il signore degli anelli, J. R. R. Tolkien: http://laboratorioteomat.weebly.com/dove-sono.html

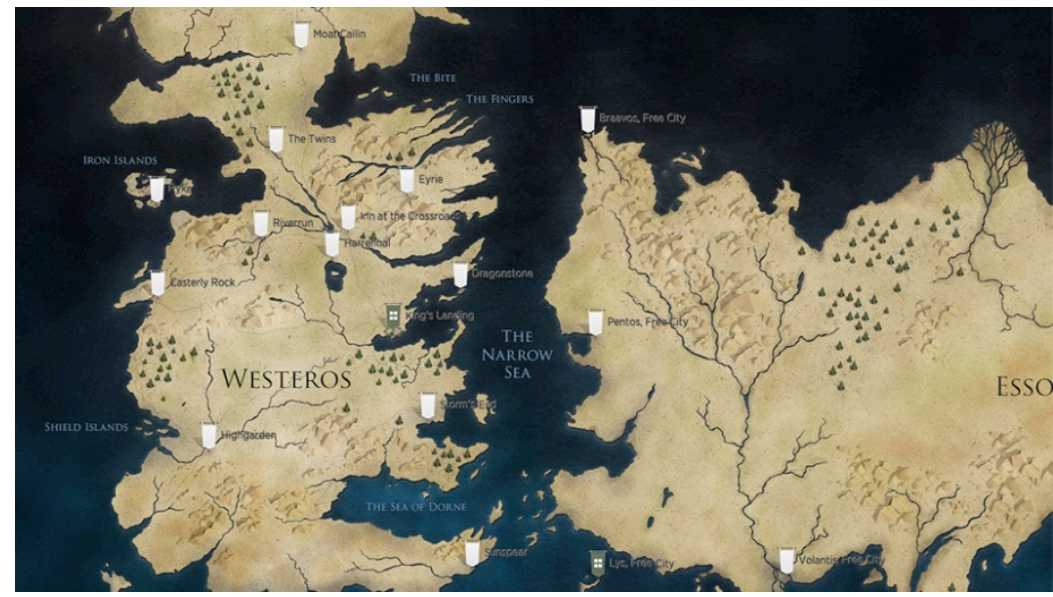

Le cronache del ghiaccio e del fuoco, G. R. R. Martin:

https://thescript.thetake.com/game-of-thrones-filming-locations-map/ 


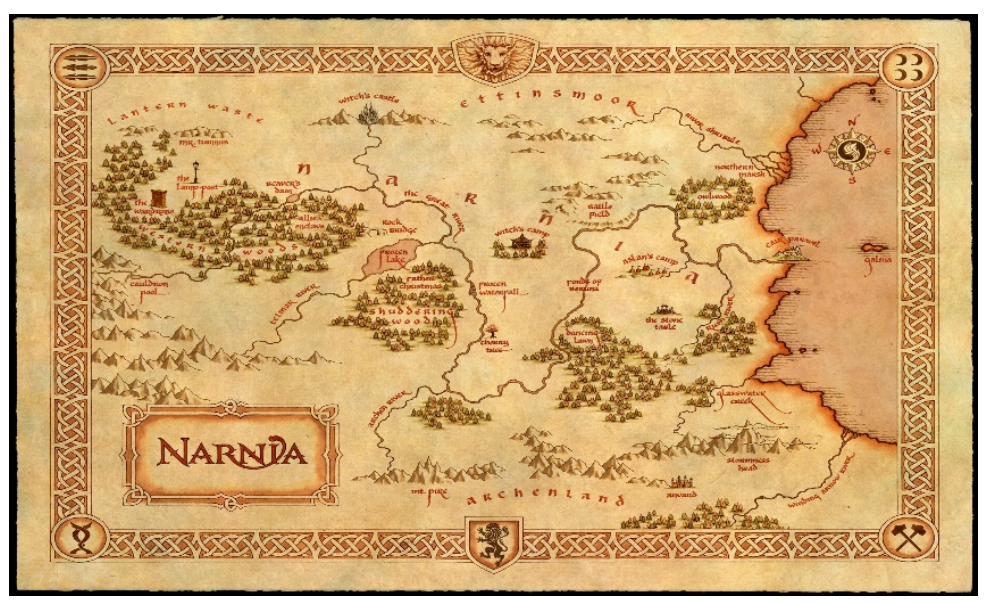

Le cronache di Narnia, C. S. Lewis:

http://www.narniaweb.com/2005/05/brand-new-narnia-map-available/

\section{Esempi di moderne carte letterarie}

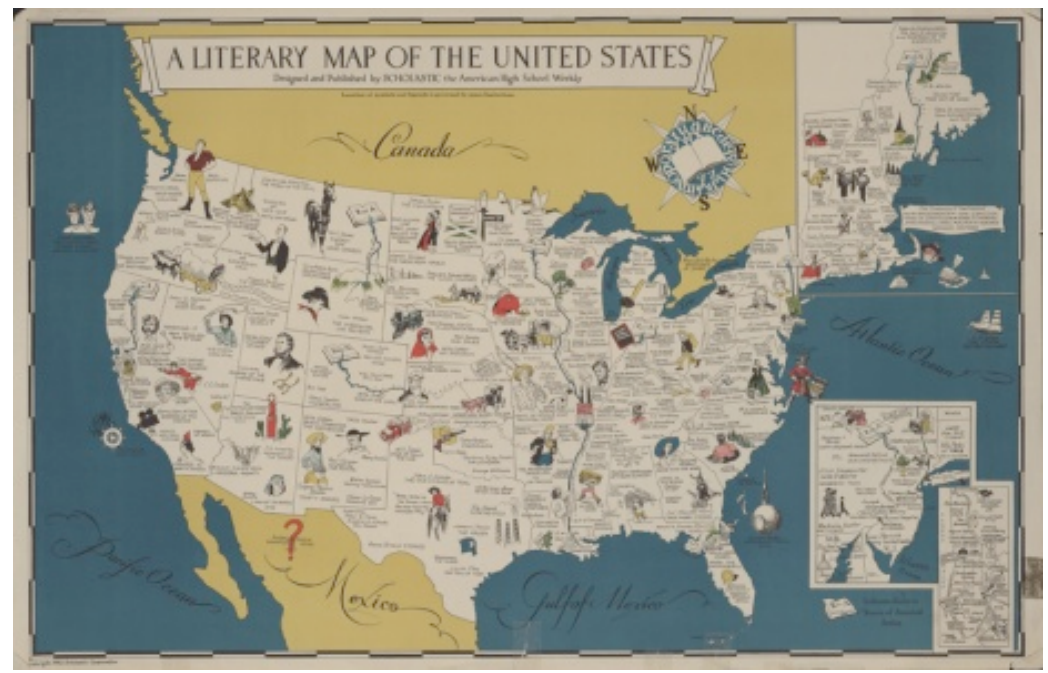

A Literary Map of the United States, 1940:

http://www.lib.umich.edu/online-exhibits/exhibits/show/litmaps/us/uslit 


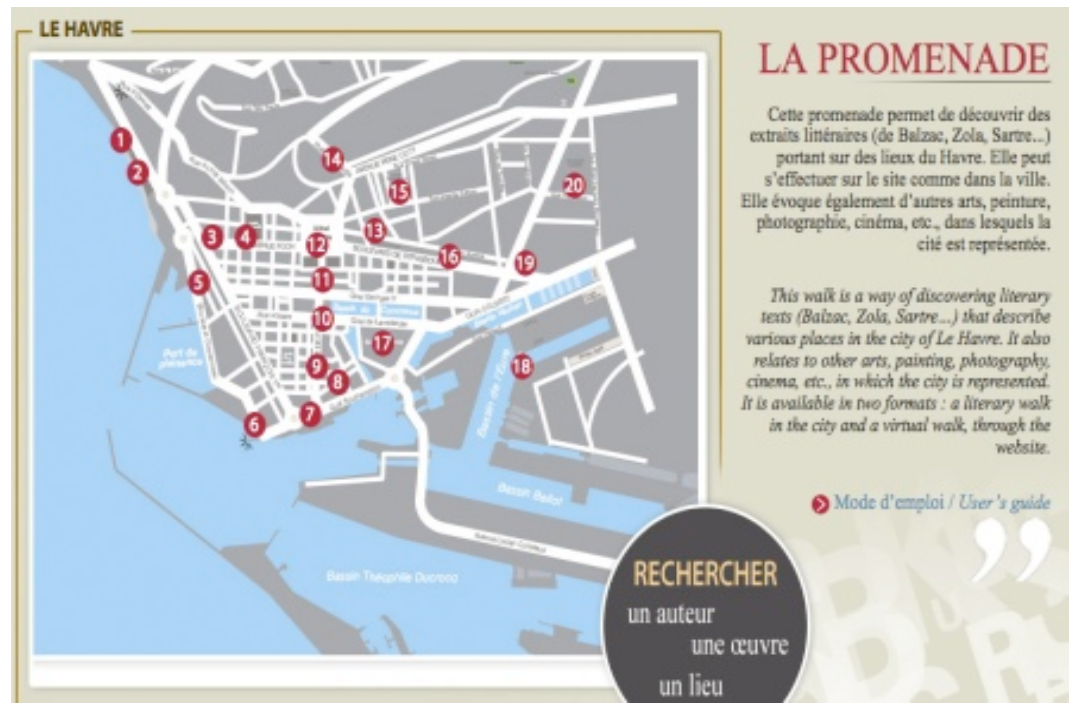

Promenade littéraire au Havre (Carte interactive de la promenade Littéraire du Havre):

https://spacefiction.wordpress.com/2015/02/09/promenade-litteraire-au-havre-literary-tour-of-le-havre/

2. 'Estate poems' $1650-1850$

Estate poems - which describe and celebrate a country estate - are most frequent in the southern counties of England where Austen's novels typically take place, while the 'Celtic periphery' is again virtually absent.

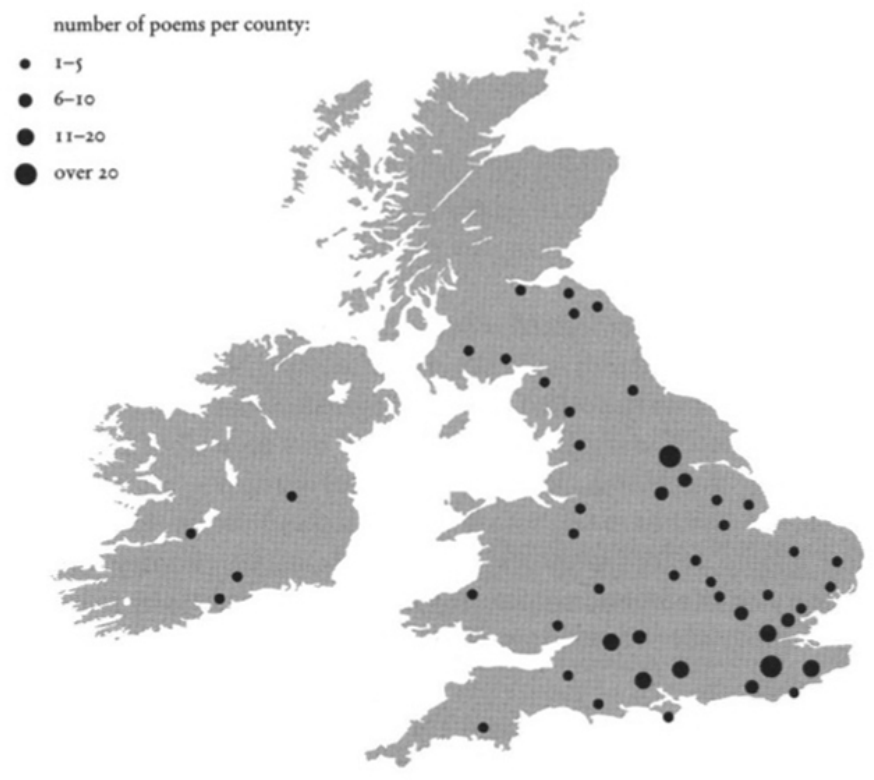

L'Inghilterra di Jane Austen:

Franco Moretti, Atlas du roman européen (1800-1900) 
Approcci interdisciplinari: letteratura e cartografia.

Tra immagini e parole.

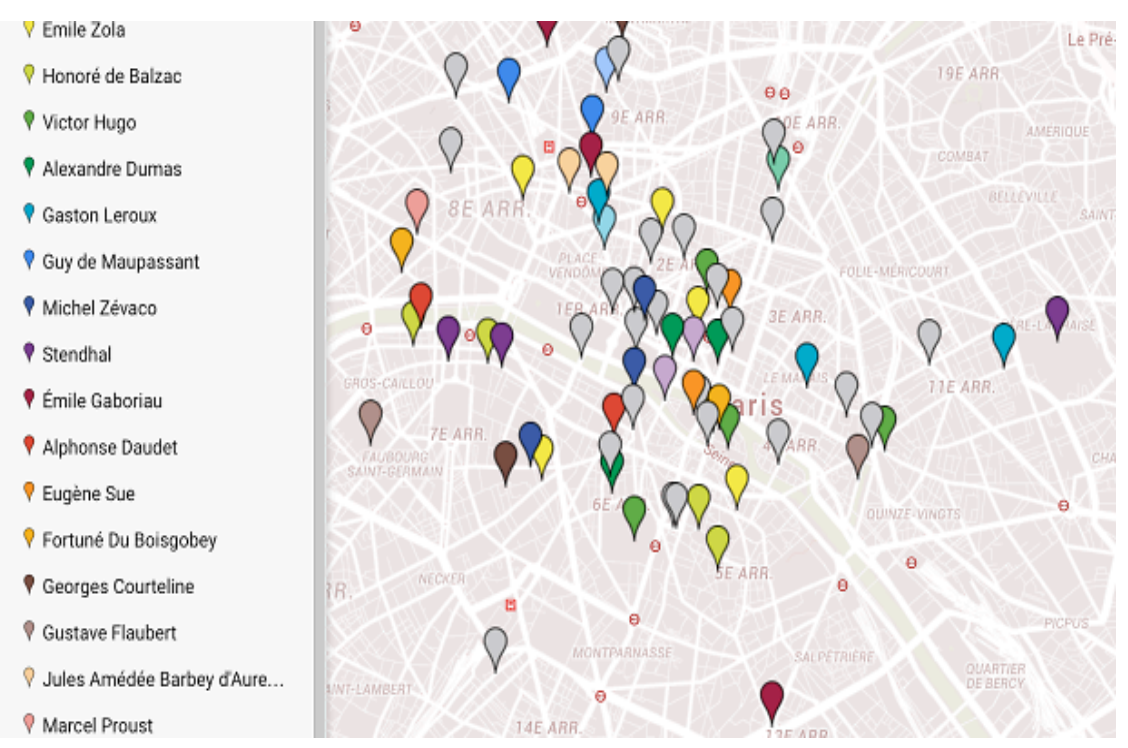

Une carte de Paris pour marcher sur les traces des classiques de la littérature:

http://www.timeout.fr/paris/le-blog/une-carte-de-paris-pour-marcher-sur-les-traces-des-classiques-dela-litterature-032516 